Cite this: Nanoscale, 2014, 6, 7052

\title{
A general mechanism for intracellular toxicity of metal-containing nanoparticles $\dagger$
}

\author{
Stefania Sabella, ${ }^{a}$ Randy P. Carney, ${ }^{b}$ Virgilio Brunetti, ${ }^{a}$ Maria Ada Malvindi, ${ }^{a}$ Noura Al- \\ Juffali, ${ }^{\text {bc }}$ Giuseppe Vecchio, ${ }^{a}$ Sam M. Janes, ${ }^{c}$ Osman M. Bakr, ${ }^{d}$ Roberto Cingolani, ${ }^{e}$ \\ Francesco Stellacci ${ }^{\star b}$ and Pier Paolo Pompa*a
}

The assessment of the risks exerted by nanoparticles is a key challenge for academic, industrial, and regulatory communities worldwide. Experimental evidence points towards significant toxicity for a range of nanoparticles both in vitro and in vivo. Worldwide efforts aim at uncovering the underlying mechanisms for this toxicity. Here, we show that the intracellular ion release elicited by the acidic conditions of the lysosomal cellular compartment - where particles are abundantly internalized - is responsible for the cascading events associated with nanoparticles-induced intracellular toxicity. We call this mechanism a "lysosome-enhanced Trojan horse effect" since, in the case of nanoparticles, the protective cellular machinery designed to degrade foreign objects is actually responsible for their toxicity. To test our hypothesis, we compare the toxicity of similar gold particles whose main difference is in the internalization pathways. We show that particles known to pass directly through cell membranes become more toxic when modified so as to be mostly internalized by endocytosis. Furthermore, using experiments with chelating and lysosomotropic agents, we found that the toxicity mechanism for

Received 6th March 2014 Accepted 6th April 2014

DOI: $10.1039 / c 4 n r 01234 h$

www.rsc.org/nanoscale different metal containing NPs (such as metallic, metal oxide, and semiconductor NPs) is mainly associated with the release of the corresponding toxic ions. Finally, we show that particles unable to release toxic ions (such as stably coated NPs, or diamond and silica NPs) are not harmful to intracellular environments.
The growing use of nanotechnology in a wide range of industrial applications is raising concerns about the safety of nanoparticles (NPs). Recent outcomes of cross-disciplinary research suggest significant toxicity of NPs both in in vitro and in vivo systems. ${ }^{1-3}$ Hence, to prevent possible harmful effects, the research community, regulatory bodies, and all the stakeholders have been recently challenged to provide a reliable risk assessment of NPs. ${ }^{4}$ Large efforts are currently devoted to understanding the main issues in nanotoxicology accounting for the potential adverse effects of NPs to humans and to the

${ }^{a}$ Istituto Italiano di Tecnologia, Center for Bio-Molecular Nanotechnologies@UniLe, Via Barsanti, 73010 Arnesano (Lecce), Italy. E-mail: pierpaolo.pompa@iit.it; Fax: +39-0832-1816230; Tel: +39-0832-1816214

${ }^{b}$ Institute of Materials, École Polytechnique Fédérale de Lausanne (EPFL), CH-1015 Lausanne, Switzerland. E-mail: francesco.stellacci@epfl.ch; Fax: +41 21 6935270; Tel: +41216937872

${ }^{c}$ Centre For Respiratory Research, Rayne Institute, University College London, 5 University Street, London WC1E 6JJ, UK

${ }^{d}$ Division of Physical Sciences and Engineering, Solar and Photovoltaics Engineering Center, King Abdullah University of Science and Technology (KAUST), Thuwal 23955-6900, Saudi Arabia

${ }^{e}$ Istituto Italiano di Tecnologia, Central Research Laboratories, Via Morego, 30-16136 Genova, Italy

$\dagger$ Electronic supplementary information (ESI) available. See DOI: $10.1039 / \mathrm{c} 4 \mathrm{nr} 01234 \mathrm{~h}$ environment. Several key findings have been made recently. Among these, it has been observed that NPs may trigger oxidative stress, ${ }^{5,6}$ inflammation, ${ }^{7}$ and indirect DNA damage ${ }^{8}$ in living systems, and that the size and shape of NPs may have a key role in determining the cellular damage. ${ }^{2,9,10}$ Moreover, it was demonstrated that the physico-chemical properties of NPs modulate their dynamic interaction with biomolecules and cellular organelles and, possibly, their toxicity., ${ }^{5,11-13}$ The formation of a biomolecular corona around the surface of NPs is a major element that defines their biological identity in biological fluids ${ }^{14-16}$ and their impact on biological functionalities. ${ }^{17}$ A protein corona may reduce cell uptake, ${ }^{12,18}$ induce protein unfolding triggering inflammation, ${ }^{7}$ or in some cases mitigate the cellular damage. ${ }^{18-21}$ It is noteworthy that some molecular mechanisms have recently emerged as new paradigms to explain NP injury, which involve lysosomal damage and autophagy. ${ }^{20,22,23}$ For instance, toxicity caused by the "proton sponge effect" has been demonstrated for cationic polymeric NPs accumulated in lysosomes. ${ }^{22}$ Interestingly, such an effect may be reduced by the presence of a protein corona around the NPs, which protects lysosomes from the bare surface of the NPs until protein degradation occurs. ${ }^{20}$ However, although much progress has been made in explaining NPs toxicity, a satisfactory integration of the various experimental 
observations as well as a general description of the mechanism underlying NPs toxicity is extremely challenging, due to the wide variety of available data, complex interactions involved, broad range of engineered NPs, and technical issues that may affect in vitro tests. ${ }^{12,24,25}$

In this work, we propose a general explanation for the toxicity of metal-containing NPs that could account for a large number of the reported observations; to the best of our knowledge, this is the first effort in this direction. We show that for a wide class of NPs (such as metallic, metal oxide, and semiconductor NPs) the acidic environment of the lysosomes triggers the release of relatively toxic ions $\left(\right.$ e.g. $, \mathrm{Ag}^{+}, \mathrm{Cd}^{2+}, \mathrm{Fe}^{2+/ 3+}$, $\mathrm{Au}^{1+/ 3+}$ ions) in the cell. We believe that these ions are the true mediators responsible for the observed intracellular toxicity profiles. Our model predicts that, once the NPs are abundantly taken-up in cells through active mechanisms of internaliza$\operatorname{tion}^{26,27}$ (i.e., the so-called Trojan horse effect), ${ }^{23,28-30}$ they can release intracellularly their cargo composed of toxic ions, as particle degradation is strongly promoted by the lysosomal environment. For this reason, we call this mechanism a "lysosome-enhanced Trojan horse effect" (LETH mechanism). This finding is quite general though counterintuitive since the cell's primary security process, engulfing and degrading the internalized foreign material via the acidic environment and proteases, instead results in overt nanoparticle toxicity. In particular, we show in a series of experiments that very similar AuNPs are significantly more toxic when entering cells via endocytosis as opposed to those mainly entering through energy independent mechanisms directly into the cytosol, and that for a large set of metal containing NPs their toxicity is mainly ascribed to their in situ degradation and intracellular release of toxic ions.

First, we investigated the time-dependent ion release of a variety of NPs, namely metallic ( $\mathrm{Au}$ and $\mathrm{Ag}$ ), magnetic $\left(\mathrm{Fe}_{3} \mathrm{O}_{4}\right)$ and semiconductor (CdSe/ZnS) NPs. The synthesis and the physico-chemical characterizations of these NPs are reported in the Methods section in the ESI. $\dagger$ The ion leakage from the NPs was assessed by inductively coupled plasma atomic emission spectroscopy (ICP-AES) under two separate conditions, mimicking either the lysosomal environment $\left(37^{\circ} \mathrm{C}, \mathrm{pH} 4.5\right)^{31,32}$ or the cellular cytoplasmic environment $\left(37^{\circ} \mathrm{C}\right.$, neutral $\left.\mathrm{pH}\right)$ (see Methods section in the ESI $\dagger$ for details). As shown in Fig. 1, for all NPs tested, we observed significant ion release in the acidic conditions and no measurable release in neutral conditions (the NP behavior in neutral conditions was also tested in cell culture medium, DMEM, 10\% FBS, pH 7.4, as a more relevant model of physiological conditions, and the same results were obtained). Such ion release was accompanied by obvious NP degradation, with consequent loss of NP morphology as well as of their fluorescence or magnetic properties (in the case of $\mathrm{CdSe} / \mathrm{ZnS}$ or $\mathrm{Fe}_{3} \mathrm{O}_{4}$, respectively, see Fig. 1, bottom) (a more detailed analysis of NP degradation in an acidic environment is reported in the ESI, Fig. S1-S4†). The ion release profiles were specific to the NPs under investigation, depending on their core material, initial concentration, and specific coating. In any case, we found that the lysosomal environment is capable of promoting NP degradation/corrosion. We hypothesized that such ions, once released intracellularly, are likely to be the main factor in promoting the toxic effects of NPs (see below).

To establish the role of lysosomal release of ions in the overall mechanism of NP toxicity, we performed several in vitro toxicity assays (i.e., viability, ROS, and caspase assays) for cells incubated with two different types of AuNPs. These particles have identical physico-chemical properties (material, size, ligand shell density, zeta potential) but a slight difference in the ligand shell composition resulting in very different surface morphologies..$^{33-35}$ The first type of particles were coated with a $2: 1$ molar mixture of 11-mercapto undecanesulfonic acid (MUS) and octanethiol (OT) and had a stripe-like morphology in their ligand shell, the second type of particles were coated with a 2 : 1 molar mixture of MUS and 2,7-dimethyl octanethiol (brOT) with a random distribution of molecules in the ligand shell. The first type of particles ("striped") were shown to pass through cell membranes mostly via an energy independent mechanism; the latter ones ("unstructured") could enter cells only by energy dependent processes, such as endocytosis. ${ }^{36,37}$ Striped and unstructured AuNPs were both tested in terms of the timedependent ion release, and showed substantially the same behavior (see Fig. $1 \mathrm{~A}$ and Fig. S5 $\dagger$ ). We evaluated the toxic effects of both AuNPs on monocytoid cells (U937), as well as on five other cell lines (see below). First, we verified that the cellular internalization of striped NPs is not affected by the inhibition of endocytotic processes, in contrast to unstructured particles that could not enter cells in these conditions (see Fig. S6†). Moreover, co-localization studies by confocal microscopy confirmed the classical lysosomal confinement of unstructured AuNPs, while striped AuNPs appear mainly distributed in the cytosol (Fig. S25 and 26†). Consistent with our toxicity model, U937 cell viability was not affected by striped AuNPs in the investigated concentration range, whilst unstructured particles caused a statistically significant time- and dose-dependent toxicity in the higher range of the investigated concentrations (Fig. 2A). Note that the striped and unstructured AuNPs were internalized in cells in similar quantities, as probed by ICP (Fig. S7†). The different toxicity behavior was further confirmed by the ROS assay, in which we observed an increase of the cellular ROS level upon treatment with unstructured NPs. Striped NPs induced ROS values close to the untreated control cells (Fig. 2B). Furthermore, as shown in Fig. 2C, we found that the unstructured AuNPs elicited a dose-dependent activation of caspase-3, whose occurrence converges on several events, including apoptosis via an intrinsic mitochondrial pathway. ${ }^{38,39}$ The same assays were also performed in other cell lines, namely human cervix carcinoma epithelial cells (HeLa), human breast adenocarcinoma epithelial cells (MCF7), human colon adenocarcinoma epithelial cells (Caco-2), human neuroblastoma cells ( $\mathrm{SH}$ SY5Y), and human hepatoma cells (Huh-7), confirming the same NP behavior (Fig. S8-S12†). Such data corroborate the hypothesis that the induced NP toxicity is internalization mechanism-dependent, namely that endocytosed NPs entrapped in the lysosomes undergo enhanced corrosion and ion leakage, with consequent toxicity to cells.

To strengthen our comparison, we focused our studies on striped nanoparticles and modified them with transferrin 
A

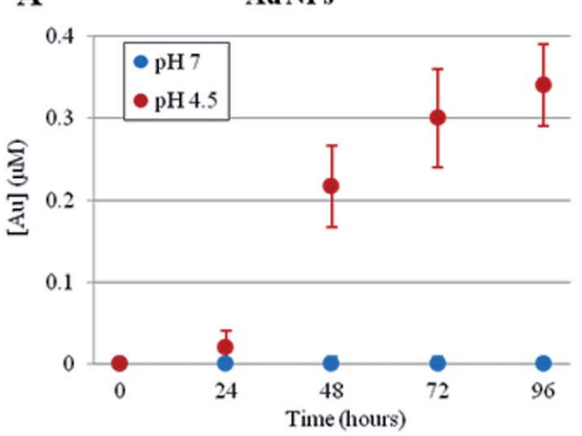

C

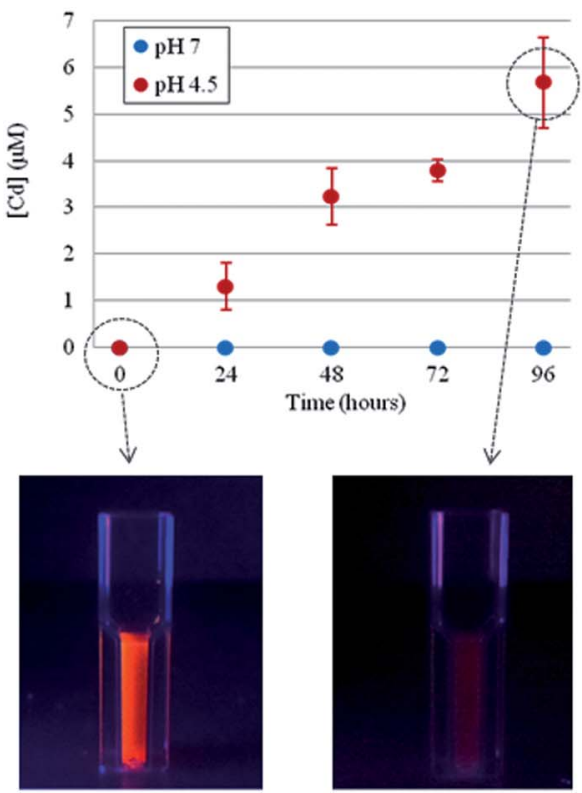

B

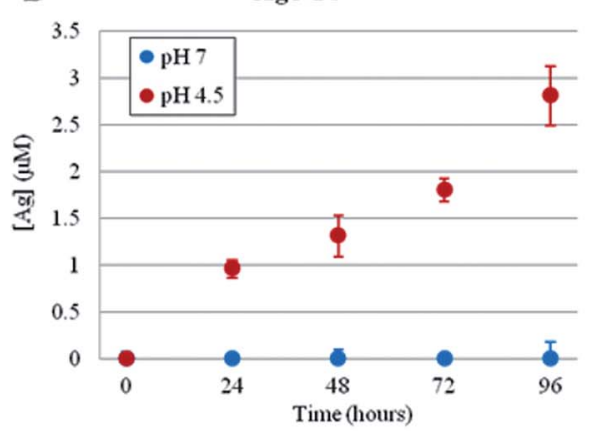

D

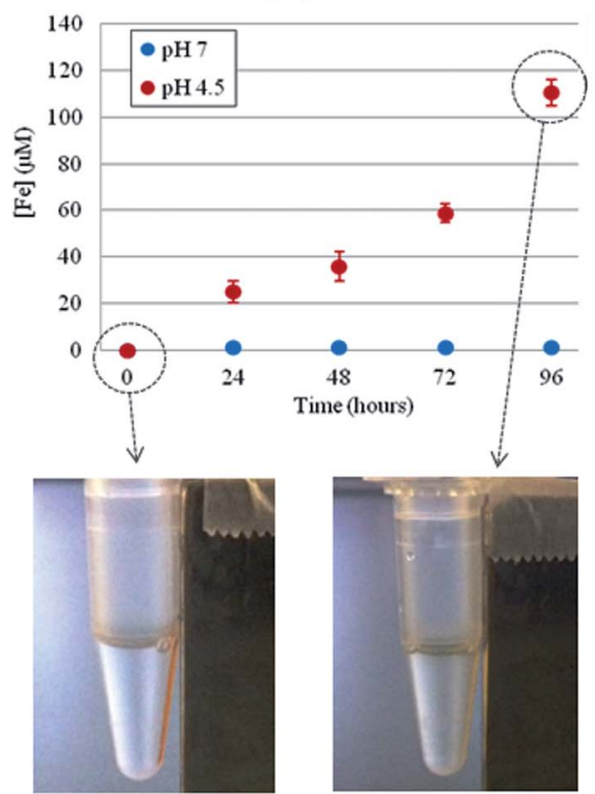

Fig. 1 Time-dependent ion release, probed by ICP-AES, of different NPs at $37^{\circ} \mathrm{C}$, in neutral (blue symbols) or acidic (red symbols) conditions. (A) $4 \mathrm{~nm}$ AuNPs, $50 \mathrm{nM}$ concentration (striped and unstructured AuNPs showed similar behavior, see also Fig. S5†); (B) $5 \mathrm{~nm}$ AgNPs, $17 \mathrm{nM}$ concentration; (C) $6 \mathrm{~nm}$ CdSe/ZnS NPs, $20 \mathrm{nM}$ concentration; (D) $10 \mathrm{~nm} \mathrm{Fe} \mathrm{O}_{4}$ NPs, $40 \mathrm{nM}$ concentration. The reported sizes of the NPs refer to their core structures (see Table S32 in the ESI $\dagger 1$ ). In (C) and (D) bottom, representative photographs of the respective NPs are also shown, at time 0 and after $96 \mathrm{~h}$ in acidic conditions, clearly revealing a significant loss of NPs' fluorescence (C) or magnetic (D) properties after the acidic treatment. Neutral and acidic conditions were obtained by dispersing the NPs in water $(\mathrm{pH} 7.0)$ or in citrate buffer ( $\mathrm{pH} 4.5)$, ${ }^{31,32}$ respectively. Neutral conditions were also probed in cell culture medium (DMEM, 10\% FBS, pH 7.4), obtaining the same results (i.e., no detectable ion release). Data represent the average from 3 independent measurements ( 6 replicates for each experiment) and the error bars indicate the standard deviation.

("Tstriped") or apolipoprotein ("Astriped"). In both cases, the intent was to modify the particles in order to activate a receptor mediated endocytosis mechanism of uptake. We incubated cells with striped, Tstriped, and Astriped NPs. We point out that the particles are exactly the same, only differing in postsynthesis functionalization (the bioconjugates characterization is reported in the ESI $\dagger$ ). Yet, only the Tstriped and Astriped particles showed significant toxicity (Fig. S13†).

The different toxicity induced by the striped and unstructured AuNPs was also verified in vivo, using the model system Drosophila melanogaster. ${ }^{\mathbf{4 0}}$ Organisms were treated with AuNPs (dose: $0.36 \mu \mathrm{g} \mathrm{g}^{-1}$ per day, see Methods section in the $\mathrm{ESI} \dagger$ for details) and their possible toxicity was evaluated by analyzing the lifespan of the different populations. Interestingly, we found that unstructured particles elicited a considerable lifespan reduction (the average lifespan, $\tau_{50}$, was $c a$. $47 \%$ lower than the control), while striped particles did not significantly affect the life cycle of the treated population (the small difference observed was not statistically significant), despite a similar bioaccumulation of the two AuNPs in the organisms (as probed by ICP measurements) (see Fig. S14 $\dagger$ ).

Overall, our model explains the toxicity of metal containing NPs in terms of intracellular release of the corresponding toxic ions. Hence, the toxicity mechanisms of NPs should follow the same molecular pathways triggered by the specific ions. In the case of AuNPs, we thus verified that the induced toxicity was mainly due to the same molecular mechanisms activated by gold ions $\left(\mathrm{Au}^{1+/ 3+}\right)$. Intracellular gold ions are known to strongly 

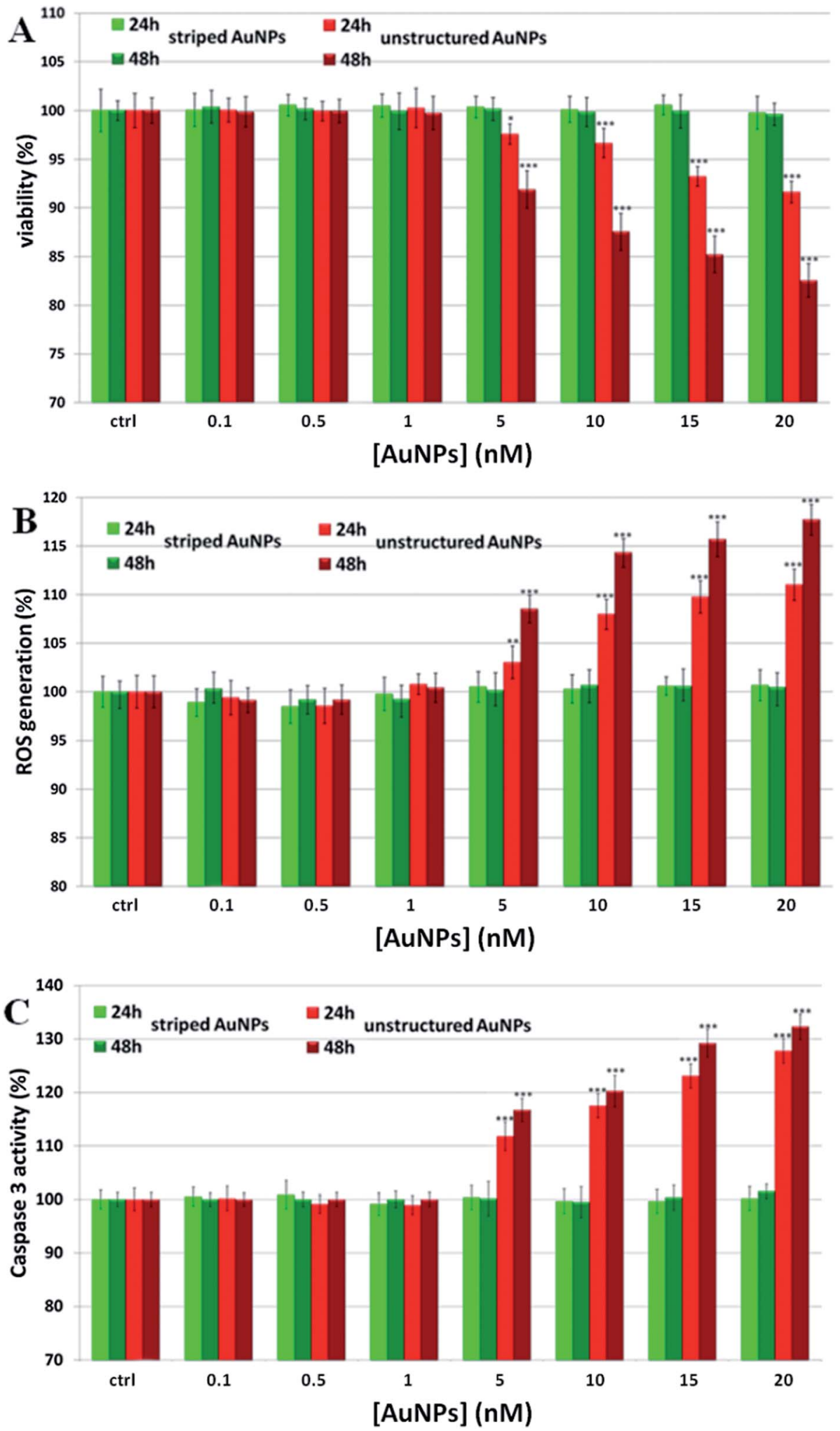

Fig. 2 Toxicity assessment of striped and unstructured AuNPs in U937 cells. (A) WST-8 proliferation assay upon treatment with increasing amount of AuNPs. Ctrl represents the negative control; values are mean \pm SD. Positive controls (not shown) were treated with $0.01 \%$ of TritonX100, displaying a strong viability decrease (ca. 80-90\%) with respect to the untreated cells. (B) ROS quantification, via DCFH-DA assay, after cellular treatment with AuNPs; values are mean $\pm \mathrm{SD}$. Positive controls (not shown) were treated with a free radical generator $\left(100 \mu \mathrm{M} \mathrm{H}_{2} \mathrm{O}_{2}\right.$ ), exhibiting a ROS increase of ca. 190-220\% with respect to the untreated control cells. (C) Evaluation of caspase 3 activity. Values are mean \pm SD. Results were analyzed by Two-way ANOVA and values compared to the control by the Bonferroni post-hoc test. Differences between treated samples and controls $(n=8)$ were considered statistically significant for $* * * P<0.001, * * P<0.01, * P<0.05$, and non-significant for $P>0.05$. 
inhibit the enzyme thioredoxin reductase (TrxR), leading to mitochondrial membrane depolarization and/or inactivation of mitochondrial enzymes ${ }^{41}$ (a more detailed description of the TrxR functionality and the impact of its inhibition on cell functions are reported in the ESI $\dagger$ ). This, in turn, causes several toxic mainstream events, including alteration of cellular redox balance, increase of physiological ROS levels, and occurrence of apoptosis. ${ }^{39}$ We verified the inhibition of TrxR by AuNPs with two experiments. First, we analyzed the inhibition of the activity of extracted TrxR by the ions released by AuNPs in both neutral and acidic environments (namely in the conditions described in Fig. 1). We observed that the Au ions released in the acidic environment are capable of reducing the TrxR activity with respect to the control ( $c a .35 \%$ of inhibition), while the negligible amount of ions generated in the neutral environment did not significantly affect the enzyme activity (Fig. 3A). This indicates that the released gold ions from the AuNPs are active against the enzyme. Furthermore, we were able to demonstrate that AuNPs can inhibit TrxR functionality directly in the cells. We analyzed the TrxR activity in HeLa cells after $48 \mathrm{~h}$ of incubation with the striped and unstructured AuNPs and also in this case, we observed that the unstructured AuNPs induced a clear reduction of the enzyme activity (Fig. 3B), at variance with the striped NPs showing a very weak enzyme inhibition. Such inhibition of TrxR functionality is consistent with the corresponding decrease in cell viability (see Fig. S15†). Analogous results were obtained by using another cell line, namely U937 (Fig. S16†). Furthermore, we found that a similar behavior, with comparable enzyme inhibition, occurs also by using citratecapped AuNPs (Fig. S17 $\dagger$ ). The available evidence indicates that the TrxR system functionality is significantly compromised by the ion cargo released in the cells by the unstructured AuNPs (see also below), caused by their lysosomal confinement. The released ions, in combination with other factors, such as the formation of peroxide intermediates (that may occur at the NP surface and/or during NP degradation ${ }^{5,42}$ ) are likely to be the main causes eliciting direct or indirect mitochondrial damage and consequent cellular redox imbalance.

The presence of different ion cargoes released intracellularly by the striped and unstructured AuNPs was quantified by measuring the amount of gold ions present in the cells upon treatment with AuNPs. As reported in Fig. 4, experimental data indicate that striped AuNPs do not release ions in the cell, whilst unstructured AuNPs, being entrapped in the lysosomes, are subjected to partial in situ degradation, leading to the release of their ion cargo (in agreement with Fig. 1). Such a cargo thus significantly contributes to NP toxicity to cells. Interestingly, these finding are also in agreement with the above data and discussion (i.e., the presence of oxidative stress and apoptosis, Fig. 2B and C) and with recent observations reporting AuNPs-induced DNA damage and genotoxicity both in vitro and in vivo. ${ }^{3,43,44}$ However, the molecular mechanism involving TrxR blocking on mitochondria (reported here as a proof of principle to show toxicity induced by released gold ions) may not be the unique target of the gold ion cargo (see also below). In fact, we cannot exclude the possibility that the same ion cargo, released by the NPs, might also be responsible for other toxic
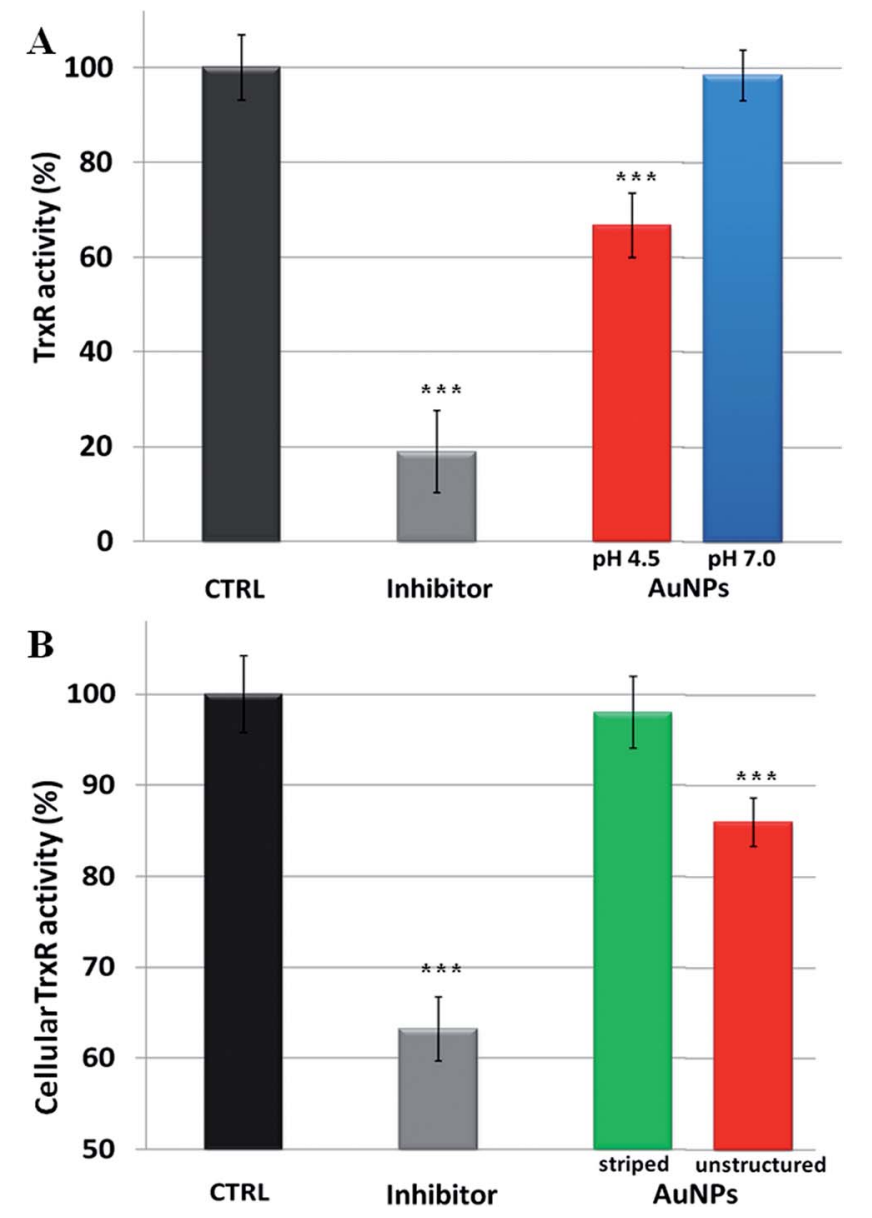

Fig. 3 Inhibition of thioredoxin reductase (TrxR) by AuNPs. (A) Inhibition of TrxR activity by the gold ions released by AuNPs at different $\mathrm{pH}$ values ( $\mathrm{pH} 4.5$ or 7.0), according to the experiment reported in Fig. 1. The inhibitor drug auranofin was used as a positive control (see Methods section in the ESI $\dagger$ ), while gold ion concentration at $\mathrm{pH} 4.5$ was $20 \mathrm{nM}$ (gold ion release was not detectable at $\mathrm{pH}$ 7.0). (B) Cellular TrxR activity in HeLa cells after $48 \mathrm{~h}$ treatment with striped and unstructured AuNPs $(15 \mathrm{nM})$ and auranofin $(1 \mu \mathrm{M})$ (see Methods section in the ESIt for experimental details). Results are mean \pm SD and differences between treated samples and controls $(n=8)$ were considered statistically significant for $* * * P<0.001$.

mechanisms, such as lysosomal (and/or RER) damage, whose dysfunction may, in turn, activate other mainstream adverse events, in line with recent findings. ${ }^{20,22,23}$

We verified our model on several types of metal containing NPs (see Table S32 $\uparrow$ for a complete list of tested NPs and their relative physical-chemical characterization), observing that the toxicity can be mainly ascribed to that of the corresponding ions. First, as shown in Fig. 5, we probed the toxicity of the four types of NPs previously tested (i.e., $\mathrm{Au}, \mathrm{Ag}, \mathrm{Fe}_{3} \mathrm{O}_{4}$, and $\mathrm{CdSe} / \mathrm{ZnS}$ NPs) upon incubation with specific ion chelators. ${ }^{45,46}$ It is noteworthy that, for all the NPs tested, the presence of the chelating agents leads to a significant reduction of the NP toxicity. This indicates a major role of intracellularly released ions in eliciting NP toxicity. When the toxic ions are chelated, the toxic effects of the NPs are minimized. Note that, in the conditions employed in these experiments, the use of chelators 


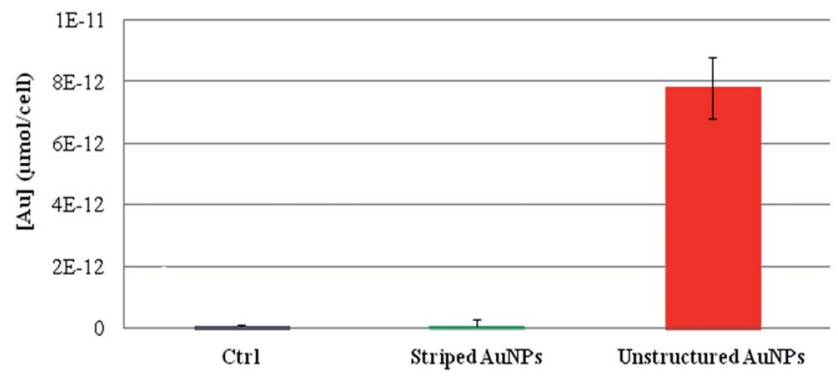

Fig. 4 ICP-AES of intracellular gold ions released by striped and unstructured AuNPs upon internalization by HeLa cells. Cells were treated with striped and unstructured AuNPs (20 nM) for 48 hours. Upon incubation, cells were lysed and ultrafiltrated by Amicon Ultra-4 (experimental details are reported in the Methods section in the ESI $\dagger$ ). The filtered solutions containing all the soluble ions were then analyzed by ICP-AES. The amount of gold ions is reported as $\mu \mathrm{mol}$ of filtered gold ions per cell. Results show that striped AuNPs do not release a measurable amount of ions, whereas unstructured AuNPs, being entrapped in the lysosomes, are subjected to partial acidic corrosion, leading to the release of their ion cargo.

did not affect the uptake efficiency of the tested NPs (see Fig. S18 ${ }^{\dagger}$ ), nor did it induce spurious off-target effects on cells (as shown in a "cross-toxicity test", Fig. S19, $\dagger$ using specific and aspecific chelating molecules). Interestingly, and in agreement with previous studies, ${ }^{22}$ the ROS level and the cellular membrane integrity have also been found to be altered by NP treatments (Fig. S20 $\dagger$ ). Yet, these toxic outcomes are also drastically reduced by the presence of chelants, further demonstrating the central role of the in situ released ions in mediating cellular toxicity (see also below).

We also tested the general applicability of our model on other metal containing NPs, namely zinc oxide, aluminum oxide, platinum, and nickel NPs (see Table S32† for their physical-chemical characterization), confirming the above data. Notably, we observed that, while all these NPs strongly reduced cell viability, treatment with the chelating agents significantly reduced (and, in some cases, completely prevented) the NP induced cellular toxicity (Fig. S21†).

To further analyze the role of the acidic environment of lysosomes as a strong enhancer of NP corrosion and consequent release of toxic ions, once the NPs are actively internalized by cells, NP induced toxicity was assessed in the presence of lysosomotropic agents, which may prevent lysosomal acidification. ${ }^{47}$ In these experiments, cells were pretreated with two lysosomotropic agents (i.e., chloroquine or ammonium chloride) enabling neutralization of lysosomal $\mathrm{pH}^{47}$ and then exposed to unstructured AuNPs or $\mathrm{Fe}_{3} \mathrm{O}_{4}$ NPs. Interestingly, as shown in Fig. 6, we observed that the cellular toxicity of both NPs was strongly attenuated by the lysosomotropic agents, thanks to the neutralization of lysosomal acidity and consequent poor release of toxic ions in the cell. This means that the same NPs, at identical doses and cellular internalization, are significantly less toxic if the intracellular release of toxic ions is prevented.
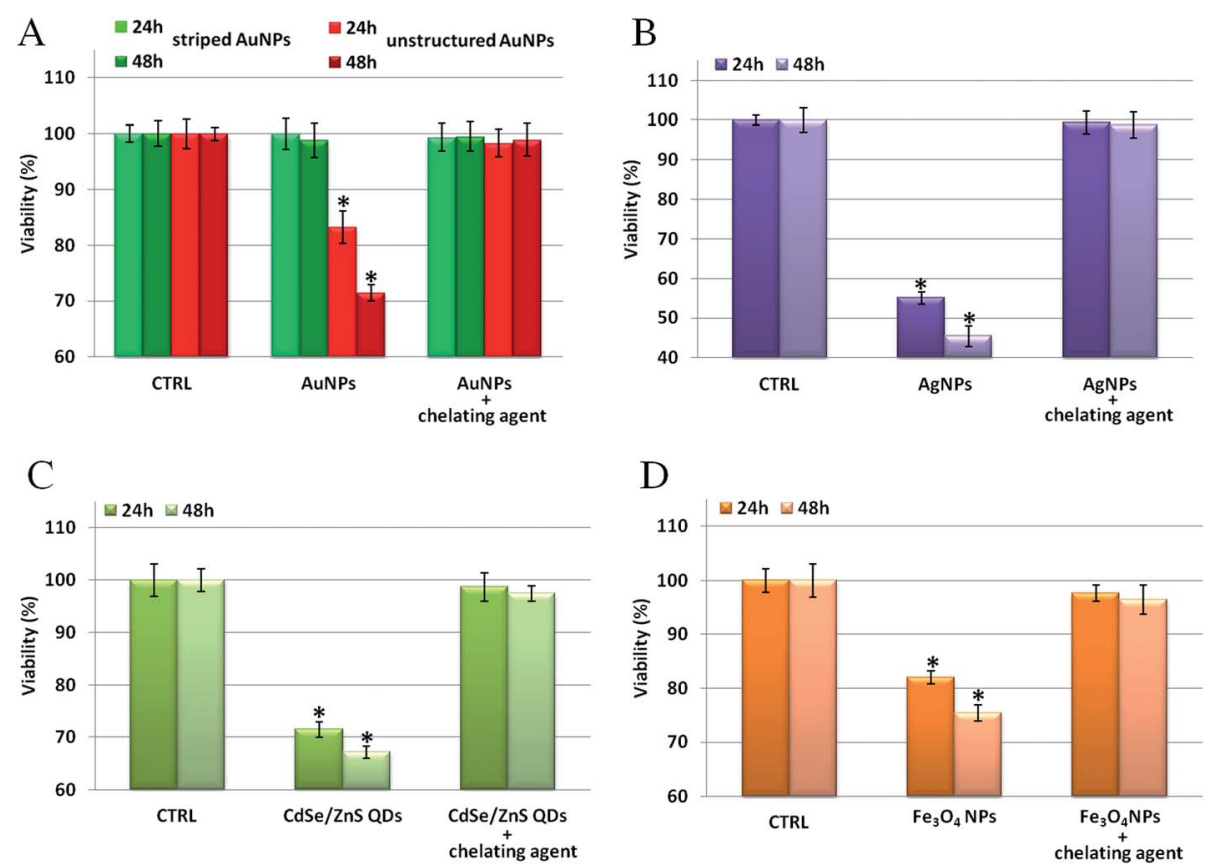

Fig. 5 Toxicity assessment of different types of NPs in the absence/presence of specific ion chelators. WST- 8 proliferation assays upon treatment with (A) striped and unstructured AuNPs (20 nM), (B) AgNPs (2 nM), and (C) CdSe/ZnS QDs (5 nM) in HeLa cells in the presence of 2,3dithiopropanol (BAL); HeLa cells were pretreated for $30 \mathrm{~min}$ with/without $1 \mu \mathrm{M} \mathrm{BAL}$ and then exposed to the NPs for 24-48 h. (D) Proliferation assay upon treatment with $2.5 \mathrm{nM}$ of $\mathrm{Fe}_{3} \mathrm{O}_{4} \mathrm{NPs}$. In this case, HeLa cells were pretreated for 30 min with/without $100 \mu \mathrm{M}$ desferrioxamine (dfx) and then exposed to $\mathrm{Fe}_{3} \mathrm{O}_{4} \mathrm{NPs}$ for 24-48 h. In all cases, the pretreatment with chelating agents suppresses, almost totally, the toxicity of the NPs. CTRL represents the negative control; values are mean \pm SD. Differences between treated samples and controls ( $n=8$ ) were considered statistically significant for $* P<0.05$ and non-significant for $P>0.05$. 

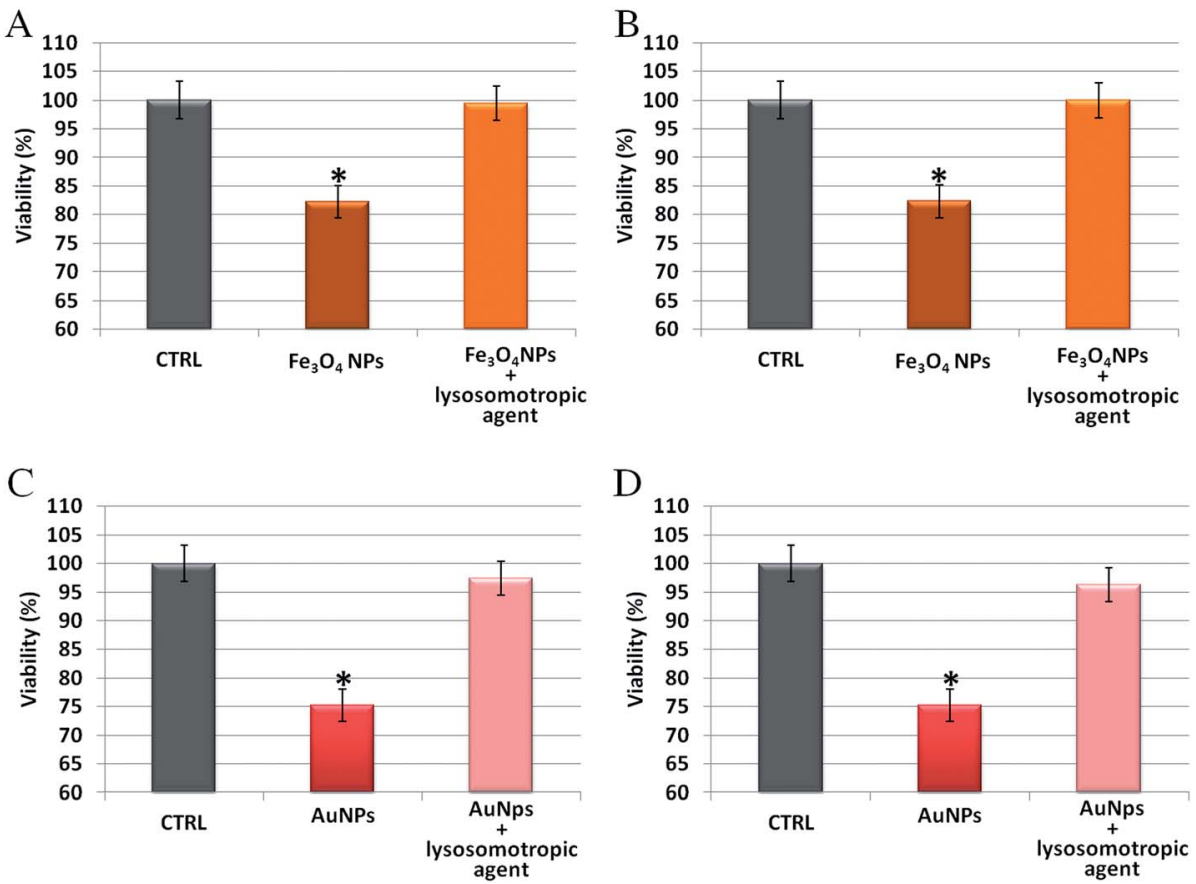

Fig. 6 Toxicity assessment of unstructured AuNPs and $\mathrm{Fe}_{3} \mathrm{O}_{4} \mathrm{NPs}$ in HeLa cells, in the presence/absence of two different lysosomotropic agents (chloroquine or ammonium chloride). (A and B) WST-8 proliferation assay upon treatment with $\mathrm{Fe}_{3} \mathrm{O}_{4} \mathrm{NPs}(2.5 \mathrm{nM})$ and (C and D) unstructured AuNPs (20 nM). HeLa cells were pretreated for $30 \mathrm{~min}$ with/without $5 \mu \mathrm{M}$ chloroquine (panels A and C) or $5 \mathrm{mM}$ ammonium chloride (panels B and D) and then exposed to the NPs for $48 \mathrm{~h}$. In all cases, NPs toxicity was strongly attenuated by the lysosomotropic agents that neutralize lysosomal acidity, preventing ion release and cytotoxicity. CTRL represents the negative control; values are mean \pm SD. Differences between treated samples and controls $(n=8)$ were considered statistically significant for $* P<0.05$ and non-significant for $P>0.05$.

It should be noted that, in terms of cellular toxicity, the Trojan horse mechanism of $\mathrm{NPs}^{23,28-30}$ is mediated and enhanced by lysosomes (see also below). NPs are abundantly internalized by cells and then lysosomes elicit the intracellular release of ions with consequent toxicity to cells. If cells are exposed to the same or even higher doses of toxic ions, dispersed in the culture medium, no toxicity is typically detected. As an example, we tested the toxicity of gold salts up to a concentration $\mathrm{ca}$. 3-fold higher than the maximum concentration used in all the cytotoxicity assays for AuNPs, and no toxicity was observed (Fig. S22 $\dagger$ ). This suggests that the ions cannot exert their toxicity because they lack the Trojan horse effect and cannot abundantly enter the cells.

We have, therefore, identified a key toxicity mechanism that we define as a "Lysosome-Enhanced Trojan Horse effect (LETH effect)" (Fig. 7). Such a mechanism may be valid for all those NPs that are actively and efficiently taken up by cells (i.e., almost all NPs $<100 \mathrm{~nm}$ ), whose acidic corrosion results in the generation of toxic ions. The LETH effect thus combines the abundant cellular internalization of the NPs via active processes with the consequent enhanced release of the relatively toxic ions (e.g., $\mathrm{Ag}^{+}, \mathrm{Cd}^{2+}, \mathrm{Fe}^{2+/ 3+}, \mathrm{Au}^{1+/ 3+}$ ions) in the cytoplasm elicited by the acidic lysosomal environment. The induced cellular toxicity can be therefore mainly ascribed to that of the corresponding ions (as also recently suggested in Cd-based QDs and ZnO $\mathrm{NPs}^{\mathbf{2 6 , 4 8 , 4 9}}$, with resulting oxidative stress and apoptosis. More generally, the significant amount of intracellularly leaked ions may exert ion-specific toxicity (e.g., enzyme inactivation as demonstrated for gold ions) against cellular targets (e.g., mitochondria, RER, etc.) and/or lysosomal damage/dysfunction, in line with recent findings with other types of NPs. ${ }^{20,22,23}$

Notably, the cellular toxicity will also depend on the physicochemical characteristics of the NPs (such as the size, nature and chemical stability of the core and ligands, surface coating, etc.), as their uptake mechanism and subsequent corrosion behavior will be a strong function of these characteristics. Also, such variables, together with protein corona effects, will affect NP uptake and their subsequent sub-cellular localization ${ }^{\mathbf{1 8 , 2 7}}$ so that NPs may encounter different microenvironments and different pHs, which, in turn, will modulate the rate of NPs degradation (and thus their toxicity). In some cases, for instance, less acidic pHs in the endo/lysosomal pathway may slow down NP degradation, mitigating the toxic effects. Such a mechanism partially accounts for the dependence of cytotoxicity on the experimental conditions (e.g., culture medium, which affects protein corona formation) as well as on the specific cell line.

A result of our study is that, in principle, it can be envisaged that the slower the lysosomal degradation of the NPs, the lower their cellular toxicity. As a consequence, we decided to apply our model to identify, by design, biocompatible nanoparticles. We selected, as a first example, our $\mathrm{Fe}_{3} \mathrm{O}_{4}$ NPs. By passivating the surface of the thin silica shell surrounding the $\mathrm{Fe}_{3} \mathrm{O}_{4}$ NPs (by functionalization with 3-(trihydroxysilyl)-1-propanesulfonic acid), we were able to reduce the ion leakage from the NPs in acidic conditions (Fig. S27A $\dagger$ ), while maintaining the original characteristics of the NPs (same size, same surface charge 


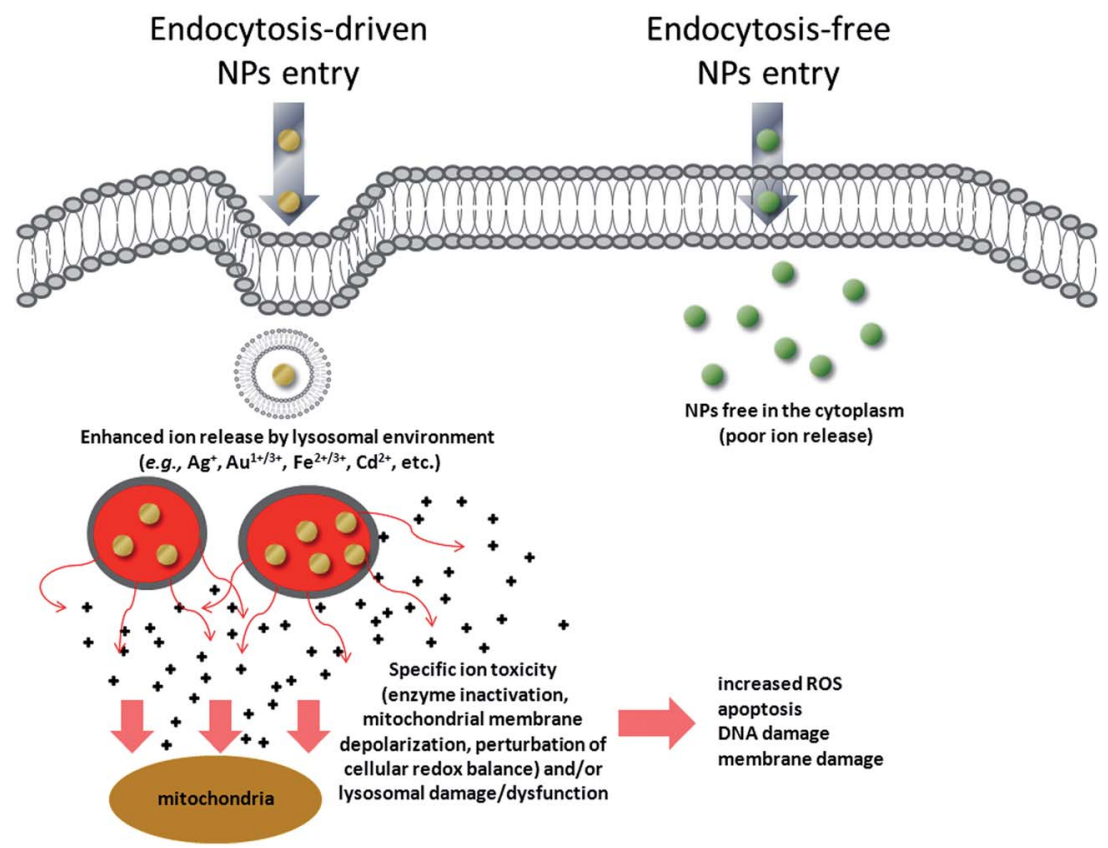

Fig. 7 Schematic of the general toxicity mechanism induced by NPs when they enter cells by active internalization mechanisms, compared to endocytosis-free NPs. NPs that enter the cells by energy-dependent processes (mediated by clathrin, caveolin, lipid raft formations and others) ${ }^{59}$ are rapidly confined in vesicular structures, endosomes, and finally in lysosomes. The acidic lysosomal pH triggers a lysosome-enhanced Trojan horse effect (LETH effect) that combines the abundant cellular internalization of the NPs via active processes with the consequent enhanced release of the relatively toxic ions (e.g., $\mathrm{Ag}^{+}, \mathrm{Cd}^{2+}, \mathrm{Fe}^{2+/ 3+}, \mathrm{Au}^{1+/ 3+}$ ions). The significant amount of intracellularly leaked ions may then exert ionspecific toxicity (e.g., enzyme depletion/inactivation, protein denaturation, etc.) against some cellular targets (e.g., mitochondria, RER) and/or lysosomal damage/dysfunction. This finally results in increased ROS levels, apoptosis, DNA and membrane damage.

$(-28 \pm 5 \mathrm{mV})$, same cellular internalization). Interestingly, such a procedure results in a remarkable reduction of cellular toxicity (Fig. S27B $\dagger$ ) as compared to the non-passivated NPs. Hence, the very same NP was found to be largely less toxic because its suitable surface engineering strongly reduces its intracellular release of toxic ions. Furthermore, we tested QDs made with a different metallic core (i.e., indium instead of cadmium), finding a similar particle degradation in acidic, lysosomal-like conditions, but no detectable impact on cellular viability or membrane integrity (Fig. S28†), as indium ions are significantly better tolerated by cells than cadmium. Moreover, as additional examples, we investigated particles that do not corrode or degrade into biocompatible components. As an example for the former, we chose diamond nanoparticles (NDs) and for the latter we chose silica particles ( $\left.\mathrm{SiO}_{2} \mathrm{NPs}\right)$. We used 5-10 nm nanodiamonds and found no measurable toxicity even using a significantly higher dose range (see Fig. S29†), in agreement with the recent literature..$^{\mathbf{5 0}, 51}$ We then moved to amorphous $\mathrm{SiO}_{2}$ NPs that, though internalized in cells via active mechanisms, were demonstrated to be non-toxic to several cell lines ${ }^{52}$ and in vivo. ${ }^{53}$ Consistently, we found that $\mathrm{SiO}_{2}$ NPs are quite resistant to acidic conditions, in which they release a low amount of silicic acid (see also Fig. S30†). ${ }^{53}$ This species is naturally found in numerous tissues and is known to be a nontoxic compound $;^{53-55}$ moreover, silicic acid administered to humans is efficiently excreted from the body through the urine. ${ }^{53}$ As a final example, we tested ceria NPs, finding no appreciable cell toxicity in the tested concentration range
(Fig. S31 $\dagger$ ). Indeed, ceria NPs have been proposed as potential candidates to fight chronic inflammation and oxidative stress, thanks to their significant antioxidant properties. It seems that nanoceria can mimic the behavior of two key antioxidant enzymes (superoxide dismutase and catalase), likely eliminating intracellular ROS via a self-regenerating mechanism. ${ }^{\mathbf{5 6}}$ Representative confocal microscopy images of lysosomal colocalization for some of the tested NPs are reported in Fig. S23S26. $\uparrow$ Overall, the LETH toxicity model opens up several routes for the development of suitable surface ligands or coatings that can prevent/reduce NP toxicity.

All the experiments performed in this work and the relative findings have been summarized in Table S37 in the ESI. $\dagger$

In conclusion, we have shown that, for a wide range of NPs (metallic, oxide, and semiconductor), the primary intracellular toxicity mechanism is common and can be explained in terms of the LETH model. Since the main cause of toxicity induced by metal containing NPs is ascribed to their corrosion in the acidic lysosomal environment, it is possible to envisage several strategies to engineer safer NPs, while maintaining their characteristic and powerful functionalities. Among such promising concepts are: (i) design of specific NP coatings to allow NPs to skip endocytotic processes; (ii) design of specific ligands or bioconjugation procedures that allow for a rapid lysosomal escape; ${ }^{57,58}$ (iii) development of robust and stable surface coatings that are resistant to the acidic $\mathrm{pH}$ of lysosomes and prevent metal ion leakage. Notably, the first two strategies are particularly attractive for nanomedicine/drug delivery applications as 
they include the additional possibility of a direct release of the NP cargo into the cell cytosol. On the other side, the third approach provides a general route, of potential industrial interest, towards the realization of biocompatible metal containing NPs. Moreover, although the LETH toxicity model described in this work cannot account for all the toxicity issues (e.g., in the case of different uptake routes of NPs such as inhalation, in the case of NP-induced inflammation, ${ }^{7}$ or in the case of environmentally dispersed NPs), it may constitute a first step toward a more detailed knowledge and categorization of the toxicity mechanisms of several classes of engineered NPs. This would also pave the way towards the development of safer nanoparticles by design and LETH-based strategies to eliminate the toxicity of metal containing NPs.

\section{Acknowledgements}

The authors gratefully acknowledge P. Kshirsagar, S. Shiv Shankar, Antonio Galeone, Gabriele Maiorano, Loris Rizzello, Georgios Anyfantis, Elisa De Luca, Hicham Chibli, Fanny Caputo, YuangFang Hou, Hakim Mehenni, and Habib Katsiev for useful discussions and help during experiments, and V. Fiorelli and B. Antonazzo for the expert technical assistance. O. M. B. and F. S. acknowledge the financial support of King Abdullah University of Science and Technology (KAUST) FIC Award (FIC/2010/02). N. A.-J. acknowledges the financial support of the Saudi Ministry of Higher Education. R. C. and F. S. acknowledge the support of the Swiss National Foundation NRP64 program.

\section{References}

1 S. Sharifi, et al. Toxicity of nanomaterials, Chem. Soc. Rev., 2012, 41, 2323-2343.

2 C. A. Poland, et al. Carbon nanotubes introduced into the abdominal cavity of mice show asbestos-like pathogenicity in a pilot study, Nat. Nanotechnol., 2008, 3, 423-428.

3 G. Vecchio, et al. Mutagenic effects of gold nanoparticles induce aberrant phenotypes in Drosophila melanogaster, $J$. Nanomed. Nanotechnol., 2012, 8, 1-7.

4 A. D. Maynard, et al. Safe handling of nanotechnology, Nature, 2006, 444, 267-269.

5 A. E. Nel, et al. Understanding biophysicochemical interactions at the nano-bio interface, Nat. Mater., 2009, 8, 543-557.

6 T. Xia, et al. Comparison of the abilities of ambient and manufactured nanoparticles to induce cellular toxicity according to an oxidative stress paradigm, Nano Lett., 2006, 6, 1794-1807.

7 Z. J. Deng, M. Liang, M. Monteiro, I. Toth and R. F. Minchin, Nanoparticle-induced unfolding of fibrinogen promotes Mac-1 receptor activation and inflammation, Nat. Nanotechnol., 2011, 6, 39-44.

8 G. Bhabra, et al. Nanoparticles can cause DNA damage across a cellular barrier, Nat. Nanotechnol., 2009, 4, 876-883.

9 K. Donaldson, F. Murphy, R. Duffin and C. Poland, Asbestos, carbon nanotubes and the pleural mesothelium: a review of the hypothesis regarding the role of long fibre retention in the parietal pleura, inflammation and mesothelioma, Part. Fibre Toxicol., 2010, 7, 5.

10 W. Jiang, Y. S. KimBetty, J. T. Rutka and C. W. ChanWarren, Nanoparticle-mediated cellular response is size-dependent, Nat. Nanotechnol., 2008, 3, 145-150.

11 M. Lundqvist, et al. Nanoparticle size and surface properties determine the protein corona with possible implications for biological impacts, Proc. Natl. Acad. Sci. U. S. A., 2008, 105, 14265-14270.

12 G. Maiorano, et al. Effects of Cell Culture Media on the Dynamic Formation of Protein-Nanoparticle Complexes and Influence on the Cellular Response, ACS Nano, 2010, 4, 7481-7491.

13 G. Oberdorster, Safety assessment for nanotechnology and nanomedicine: concepts of nanotoxicology, J. Intern. Med., 2010, 267, 89-105.

$14 \mathrm{~T}$. Cedervall, et al. Understanding the nanoparticle-protein corona using methods to quantify exchange rates and affinities of proteins for nanoparticles, Proc. Natl. Acad. Sci. U. S. A., 2007, 104, 2050-2055.

15 D. Walczyk, F. B. Bombelli, M. P. Monopoli, I. Lynch and K. A. Dawson, What the cell "sees" in bionanoscience, $J$. Am. Chem. Soc., 2010, 132, 5761-5768.

16 P. Aggarwal, J. B. Hall, C. B. McLeland, M. A. Dobrovolskaia and S. E. McNeil, Nanoparticle interaction with plasma proteins as it relates to particle biodistribution, biocompatibility and therapeutic efficacy, Adv. Drug Delivery Rev., 2009, 61, 428-437.

17 M. P. Monopoli, C. Aberg, A. Salvati and K. A. Dawson, Biomolecular coronas provide the biological identity of nanosized materials, Nat. Nanotechnol., 2012, 7, 779-786.

18 A. Lesniak, et al. Effects of the presence or absence of a protein corona on silica nanoparticle uptake and impact on cells, ACS Nano, 2012, 6, 5845-5857.

$19 \mathrm{C}$. Ge, et al. Binding of blood proteins to carbon nanotubes reduces cytotoxicity, Proc. Natl. Acad. Sci. U. S. A., 2011, 108, 16968-16973.

$20 \mathrm{~F}$. Wang, et al. The biomolecular corona is retained during nanoparticle uptake and protects the cells from the damage induced by cationic nanoparticles until degraded in the lysosomes, Nanomedicine, 2013, 9, 1159-1168.

$21 \mathrm{~W}$. Hu, et al. Protein corona-mediated mitigation of cytotoxicity of graphene oxide, ACS Nano, 2011, 5, 3693-3700.

22 T. Xia, M. Kovochich, M. Liong, J. I. Zink and A. E. Nel, Cationic polystyrene nanosphere toxicity depends on cellspecific endocytic and mitochondrial injury pathways, ACS Nano, 2008, 2, 85-96.

23 S. T. Stern, P. P. Adiseshaiah and R. M. Crist, Autophagy and lysosomal dysfunction as emerging mechanisms of nanomaterial toxicity, Part. Fibre Toxicol., 2012, 9, 20.

24 E. C. Cho, Q. Zhang and Y. Xia, The effect of sedimentation and diffusion on cellular uptake of gold nanoparticles, Nat. Nanotechnol., 2011, 6, 385-391.

25 J. A. Kim, C. Aberg, A. Salvati and K. A. Dawson, Role of cell cycle on the cellular uptake and dilution of nanoparticles in a cell population, Nat. Nanotechnol., 2012, 7, 62-68. 
26 S. J. Soenen, et al. Cellular toxicity of inorganic nanoparticles: Common aspects and guidelines for improved nanotoxicity evaluation, Nano Today, 2011, 6, 446-465.

27 T.-G. Iversen, T. Skotland and K. Sandvig, Endocytosis and intracellular transport of nanoparticles: Present knowledge and need for future studies, Nano Today, 2011, 6, 176-185.

28 R. F. Service, Nanotechnology. Nanoparticle Trojan horses gallop from the lab into the clinic, Science, 2010, 330, 314315.

29 J. F. Kukowska-Latallo, et al. Nanoparticle targeting of anticancer drug improves therapeutic response in animal model of human epithelial cancer, Cancer Res., 2005, 65, 5317-5324.

30 E. J. Park, J. Yi, Y. Kim, K. Choi and K. Park, Silver nanoparticles induce cytotoxicity by a Trojan-horse type mechanism, Toxicol. In Vitro, 2010, 24, 872-878.

31 A. S. Arbab, et al. A model of lysosomal metabolism of dextran coated superparamagnetic iron oxide (SPIO) nanoparticles: implications for cellular magnetic resonance imaging, NMR Biomed., 2005, 18, 383-389.

32 M. Lévy, et al. Degradability of superparamagnetic nanoparticles in a model of intracellular environment: follow-up of magnetic, structural and chemical properties, Nanotechnology, 2010, 21, 395103.

33 A. M. Jackson, J. W. Myerson and F. Stellacci, Spontaneous assembly of subnanometre-ordered domains in the ligand shell of monolayer-protected nanoparticles, Nat. Mater., 2004, 3, 330-336.

34 Q. K. Ong, et al. High-resolution scanning tunneling microscopy characterization of mixed monolayer protected gold nanoparticles, ACS Nano, 2013, 7, 8529-8539.

35 F. Biscarini, et al. Quantitative Analysis of Scanning Tunneling Microscopy Images of Mixed-Ligand Functionalized Nanoparticles, Langmuir, 2013, 29, 1372313734.

36 A. Verma, et al. Surface-structure-regulated cell-membrane penetration by monolayer-protected nanoparticles, Nat. Mater., 2008, 7, 588-595.

37 R. C. Van Lehn, et al. Effect of particle diameter and surface composition on the spontaneous fusion of monolayerprotected gold nanoparticles with lipid bilayers, Nano Lett., 2013, 13, 4060-4067.

38 A. G. Porter and R. U. Janicke, Emerging roles of caspase-3 in apoptosis, Cell Death Differ., 1999, 6, 99-104.

39 S. W. G. Tait and D. R. Green, Mitochondria and cell death: outer membrane permeabilization and beyond, Nat. Rev. Mol. Cell Biol., 2010, 11, 621-632.

40 B. H. Jennings, Drosophila - a versatile model in biology \& medicine, Mater. Today, 2011, 14, 190-195.

41 P. J. Barnard and S. J. Berners-Price, Targeting the mitochondrial cell death pathway with gold compounds, Coord. Chem. Rev., 2007, 251, 1889-1902.
$42 \mathrm{~J}$. Liu and R. H. Hurt, Ion release kinetics and particle persistence in aqueous nano-silver colloids, Environ. Sci. Technol., 2010, 44, 2169-2175.

43 W.-S. Cho, et al. Acute toxicity and pharmacokinetics of 13 nm-sized PEG-coated gold nanoparticles, Toxicol. Appl. Pharmacol., 2009, 236, 16-24.

44 S. Sabella, et al. Toxicity of citrate-capped AuNPs: an in vitro and in vivo assessment, J. Nanopart. Res., 2011, 13, 68216835.

45 S. J. S. Flora and V. Pachauri, Chelation in Metal Intoxication, Int. J. Environ. Res. Public Health, 2010, 7, 2745-2788.

46 S. J. H. Soenen, U. Himmelreich, N. Nuytten and M. De Cuyper, Cytotoxic effects of iron oxide nanoparticles and implications for safety in cell labelling, Biomaterials, 2011, 32, 195-205.

47 U. A. Ashfaq, T. Javed, S. Rehman, Z. Nawaz and S. Riazuddin, Lysosomotropic agents as HCV entry inhibitors, Virol. J., 2011, 8, 163.

48 G. Gobe and D. Crane, Mitochondria, reactive oxygen species and cadmium toxicity in the kidney, Toxicol. Lett., 2010, 198, 49-55.

49 W.-S. Cho, et al. Progressive severe lung injury by zinc oxide nanoparticles; the role of $\mathrm{Zn}^{2+}$ dissolution inside lysosomes, Part. Fibre Toxicol., 2011, 8, 27.

50 E. K. Chow, et al. Nanodiamond Therapeutic Delivery Agents Mediate Enhanced Chemoresistant Tumor Treatment, Sci. Transl. Med., 2011, 3, 73ra21.

51 V. N. Mochalin, O. Shenderova, D. Ho and Y. Gogotsi, The properties and applications of nanodiamonds, Nat. Nanotechnol., 2012, 7, 11-23.

52 M. A. Malvindi, et al. $\mathrm{SiO}_{2}$ nanoparticles biocompatibility and their potential for gene delivery and silencing, Nanoscale, 2012, 4, 486-495.

53 J. H. Park, et al. Biodegradable luminescent porous silicon nanoparticles for in vivo applications, Nat. Mater., 2009, 8, 331-336.

54 L. M. Bimbo, et al. Biocompatibility of Thermally Hydrocarbonized Porous Silicon Nanoparticles and their Biodistribution in Rats, ACS Nano, 2010, 4, 3023-3032.

55 S. P. Low, N. H. Voelcker, L. T. Canham and K. A. Williams, The biocompatibility of porous silicon in tissues of the eye, Biomaterials, 2009, 30, 2873-2880.

56 I. Celardo, J. Z. Pedersen, E. Traversa and L. Ghibelli, Pharmacological potential of cerium oxide nanoparticles, Nanoscale, 2011, 3, 1411-1420.

57 E. Oh, et al. Cellular Uptake and Fate of PEGylated Gold Nanoparticles Is Dependent on Both Cell-Penetration Peptides and Particle Size, ACS Nano, 2011, 5, 6434-6448.

58 K. T. Thurn, et al. Nanoparticles for applications in cellular imaging, Nanoscale Res. Lett., 2007, 2, 430-441.

59 S. D. Conner and S. L. Schmid, Regulated portals of entry into the cell, Nature, 2003, 422, 37-44. 